\title{
The Contributing Risk of Tobacco Use for ARDS Development in Burn-Injured Adults With Inhalation Injury
}

\author{
Majid Afshar MD MSc, Giora Netzer MD MSc, Michael J Mosier MD, Richard S Cooper MD, \\ William Adams MSc, Ellen L Burnham MD, Elizabeth J Kovacs PhD, \\ Ramon Durazo-Arvizu PhD, and Stephanie Kliethermes PhD
}

\begin{abstract}
BACKGROUND: This study aims to determine the relationship between tobacco use, inhalation injury, and ARDS in burn-injured adults. METHODS: This study was an observational cohort of 2,485 primary burn admissions to a referral burn center between January 1, 2008 and March 15, 2015. Subjects were evaluated by methods used to account for mediation and traditional approaches (multivariable logistic regression and propensity score analysis). Mediation analysis examined both the (1) indirect effect of tobacco use via inhalation injury as the mediator on ARDS development and (2) the direct effect of tobacco use alone on ARDS development. RESULTS: ARDS development occurred in $6.8 \%(n=170)$ of the cohort. Inhalation injury occurred in $5.0 \%(n=125)$ of the cohort, and ARDS developed in $48.8 \%(n=83)$ of the subjects with inhalation injury. Tobacco use was 2-fold more common in subjects with ARDS. In the mediated model, the direct effect of tobacco use on ARDS, including interaction between tobacco use and inhalation injury, was not significant (odds ratio [OR] 1.63, 95\% CI 0.91-2.92, $P=.10$ ). However, the indirect effect of tobacco use via inhalation injury as the mediator was significant $(O R$ 1.61, 95\% CI 1.25-2.07, $P<.001$ ), and the proportion of the total effect of tobacco use operating through the mediator was $55.6 \%$. In the non-mediation models (multivariable logistic regression and propensity score analysis), which controlled for inhalation injury and other covariables, the OR for the association between tobacco use and ARDS was $1.84(95 \% \mathrm{CI} 1.22-2.81, P<.001)$ and $1.69(95 \% \mathrm{CI}$ $1.04-2.75, P=.03$ ), respectively. CONCLUSIONS: In mediation analysis, inhalation injury was the overwhelming predictor for ARDS development, whereas tobacco use has its strongest effect indirectly through inhalation injury. Patients with at least moderate inhalation injury are at greatest risk for ARDS development despite baseline risk factors like tobacco use. Key words: ARDS; acute lung injury; inhalation injury; burn injury; tobacco use. [Respir Care 2017;62(11):1456-1465. (C) 2017 Daedalus Enterprises]
\end{abstract}

\section{Introduction}

More than one million burn injuries occur yearly in the United States, and survivorship is worse when the injury is

Drs Afshar and Mosier are affiliated with the Burn and Shock Trauma Research Institute, and Drs Afshar, Cooper, and Durazo-Arvizu and $\mathrm{Mr}$ Adams are affiliated with the Department of Public Health Sciences, Stritch School of Medicine, Loyola University Health Sciences Campus, Maywood, Illinois. Dr Afshar is also affiliated with the Division of Pulmonary and Critical Care Medicine, Loyola University Chicago, Maywood, Illinois. Dr Netzer is affiliated with the Division of Pulmonary and Critical Care Medicine, University of Maryland, Baltimore Maryland. Dr Burnham is affiliated with the Division of Pulmonary Sciences and Crit- associated with inhalation injury. ${ }^{1}$ Prior epidemiologic studies have shown that nearly half of residential fires and fatalities are associated with cigarettes or combustible tobacco products, and house fires involving cigarettes ac- 
count for approximately one third of fire fatalities in the United States..$^{2-4}$ Internationally, smoking is the leading cause of residential or total fire death and is an independent risk factor for thermal injury. ${ }^{4,5}$ Inhalation injury accounts for approximately $5 \%$ of in-patient admissions for burns. ${ }^{6}$ Burn care has improved in recent years, and mortality in burn injuries with total body surface area between 30 and $39 \%$ was $16 \%$ in $2013 .{ }^{1}$ However, morbidity from organ dysfunction, such as ARDS, remains poorly characterized.

Patient cohorts have demonstrated that $>25 \%$ of subjects with large burns or inhalation injuries who survive their injury develop ARDS. ${ }^{7,8}$ Cohort studies to examine important risk factors for ARDS in the setting of burn injury have been limited by small sample sizes or by including only military personnel.9,10 Risk prediction models that have incorporated tobacco use as a predictor in patients are mainly derived from medical cohorts with sepsis and pneumonia. ${ }^{7}$ Both active and passive tobacco use are independent risk factors for the development of ARDS in critically ill patients, including trauma patients. ${ }^{11-13}$ However, in burn injury, tobacco use commonly precedes the burn injury and can have both a direct effect on ARDS and an indirect effect on ARDS through the burn injury it preceded. Separating tobacco's direct effect on ARDS versus its indirect effects via other lung injury mediators (eg, inhalation injury) cannot be fully evaluated with traditional statistical methods that do not account for the temporality in the pathway between tobacco use, inhalation injury, and ARDS. Mediation analysis, a method adopted from social sciences, allows for evaluation of simultaneous regression pathways on an outcome. This method was employed to examine the impact of driving pressures within a lung-protective ventilation strategy that improved mortality in patients with ARDS. ${ }^{14}$

To further clarify the association between tobacco use, inhalation injury, and development of ARDS, we examined subjects from a high-volume regional referral burn center and burn research institute during a 7-y period. We hypothesized that burn injury itself was the

This research was supported in part by National Institute of Alcoholism and Alcohol Abuse grants K23AA024503 (to MA) and R21 AA023193 (to EJK) and National Institute of General Medical Sciences grant R01 GM115257 (to EJK). The authors have disclosed no conflicts of interest.

Supplementary material related to this paper is available at http:// www.rcjournal.com.

Correspondence: Majid Afshar MD MSc, Loyola University Chicago, Center for Translational Research and Education, Room 447, 2160 South First Avenue, Maywood, IL 60153. E-mail: majid.afshar@luhs.org.

DOI: $10.4187 /$ respcare. 05560

\section{QUICK LOOK}

\section{Current knowledge}

Prior studies examining tobacco use have identified it as a risk factor for ARDS in other critically ill patients. However, burn injury is less clear and requires analysis into the temporal relationship because smoking is an independent risk factor for inhalation injury and confers risk for ARDS through inhalation injury.

\section{What this paper contributes to our knowledge}

Inhalation injury was an overwhelming predictor for ARDS, with $56 \%$ of the increased risk due to inhalation injury as the mediator. This was observed despite interactions between tobacco use and inhalation injury as well as adjustment for total body surface area, mechanism of injury, and subject characteristics. Prospective clinical studies are needed to better examine the effect of inhalation injury over other risk factors so that clinicians may focus care to attenuate the strongest determinants for ARDS development.

overwhelming predictor of ARDS, and the risk contributed by tobacco use was not significant. We examined our hypothesis using traditional statistical approaches as well as mediation analysis to compare how tobacco use is associated with ARDS with and without inhalation injury as the mediator.

\section{Methods}

We analyzed data from an observational cohort of consecutive burn-injured adults admitted between January 1, 2008 and March 15, 2015 to Loyola University Chicago Burn Center. Loyola University Chicago is a tertiary academic center and referral burn center treating nearly 700 patients annually. All patients admitted to the burn center were recorded in the institution's burn registry and maintained within the Burn and Shock Trauma Research Institute. All admissions, burn injury characteristics, and dates of burn injury were verified by 2 full-time clinical research nurses in the burn unit with $>15 \mathrm{y}$ of dedicated clinical and research experience. Additional clinical variables were extracted from the electronic medical record by linkage of the burn registry to the clinical research database.

\section{Subject Selection and Main Outcome Measure}

Patients $\geq 18$ y of age and with a primary admission for burn were evaluated. Patients admitted for non-burn inju- 
ries and subsequent encounters for prior injuries were excluded. The primary exposure of interest, tobacco use disorder, was defined using administrative data for tobacco use from screening intake questionnaire data or claims data. Subject comorbidities, such as diabetes mellitus type I or II, hypertension, any respiratory disease, and congestive heart failure, were collected from administrative data. Percentage of total body surface area injured was verified after subject discharge by review of operative notes and attending documentation from the clinical research nurses. All subjects receiving invasive mechanical ventilation with suspected inhalation injury received a fiberoptic bronchoscopic examination to evaluate for severity of injury. During bronchoscopy, inhalation injury was graded based on abbreviated injury score. ${ }^{15}$

\section{ARDS}

The Berlin definition ${ }^{16}$ was applied via an automated electronic screening tool for identification of ARDS cases. The authors designed the tool in a structured query language-based integrative database from the clinical research database. Electronic medical records served as the main data source for the rules of development with linked mechanical ventilation data, arterial blood gas data, and chest imaging reports. The tool was adapted and updated from prior internal and external validation studies in trauma subjects using a rule-based keyword search approach. ${ }^{17}$ The authors have previously applied this tool in other trauma registries. ${ }^{18}$ In a simple random sample of $50 \%$ of the patients with inhalation injury from our burn registry, we demonstrated similar test characteristics with a sensitivity and specificity of 76.4 and $88 \%$ after review of the chest radiograph images by a pulmonary and critical care physician (MA). The approach adapted to the Berlin definition is shown in the Appendix (see the supplementary materials at http://www.rcjournal.com).

\section{Covariables}

Candidate variables were identified a priori as risk factors for ARDS, , $, 11,19-21$ and their selection for possible inclusion in the adjusted model was made if they had a $P$ value $<.20$ in univariable analysis. Candidate variables included age, sex, race, burn mechanism, tobacco use, diabetes, inhalation injury, total body surface area injured, and burn mechanism. Alcohol use was not evaluated for inclusion into the multivariable models due to a high proportion of missing data. Inhalation injury was categorized as a dichotomous variable (not indicated/abbreviated injury score $=0$ vs abbreviated injury score $\geq 1$ ). Total body surface area injury at $33 \%$ demonstrated the optimal cutoff for development of ARDS using receiver operating characteristic area under the curve with a sensitivity and spec- ificity of 65.3 and $88.9 \%$, respectively. Therefore, total body surface area injured was evaluated as a dichotomous variable at $\geq 33 \%$. Burn mechanism was a dichotomous variable as flame versus non-flame because flame mechanism comprised $45.3 \%$ of the cohort. Due to the low rates of comorbidities, respiratory diseases were combined. The small sample size of any individual respiratory disease did not allow for examination of the role of different respiratory diseases on outcomes.

\section{Analysis}

In univariable analysis, continuous variables were evaluated as medians and interquartile ranges and analyzed using either Wilcoxon rank sum or Kruskal-Wallis nonparametric tests, whereas categorical variables were analyzed using a chi-square test or Fisher exact test, if appropriate. Three different models were used to examine the association between tobacco use and development of ARDS. In the first model, multivariable logistic regression was used to assess the association (odds ratio) between tobacco use and ARDS development with adjustment for age, sex, mechanism of burn, total body surface area injured, and inhalation injury. In the second model, 2:1 matched propensity score analysis was performed, with age, sex, mechanism of burn, total body surface area injured, and inhalation injury used to create the propensity. Using each matched set as a stratum, a conditional logistic regression model was applied to estimate the association of tobacco use and ARDS. In the third model, a pathway framework was used to decompose the total effect of tobacco use on ARDS and incorporate inhalation injury as the mediating effect. ${ }^{22}$ This included the effect of tobacco directly on ARDS (pure direct effect). A second pathway was the indirect effect of tobacco on ARDS through the mediator inhalation injury (pure indirect effect). These pathways included analysis that allowed for exposuremediator (tobacco-inhalation injury) interaction, referred to as the total direct and total indirect effect, respectively (Fig. 1). The model also allowed for both exposure-outcome confounders (age, sex, total body surface area injured) and mediator-outcome confounders (burn mechanism, total body surface area injured). Direct and indirect effects on the odds ratio scale was estimated via this approach. We used 500 bootstrap samples and the percentile method to produce $95 \%$ CIs. The same mediation analysis was performed for the flame subgroup, which is the predominant mechanism of burn injury. Other important mediator-outcome confounders, such as sepsis, have been shown to occur frequently in critically ill burn patients, so sensitivity analysis was performed for unmeasured confounding. All statistical analyses were performed using SAS 9.4 (SAS Institute, Cary, 


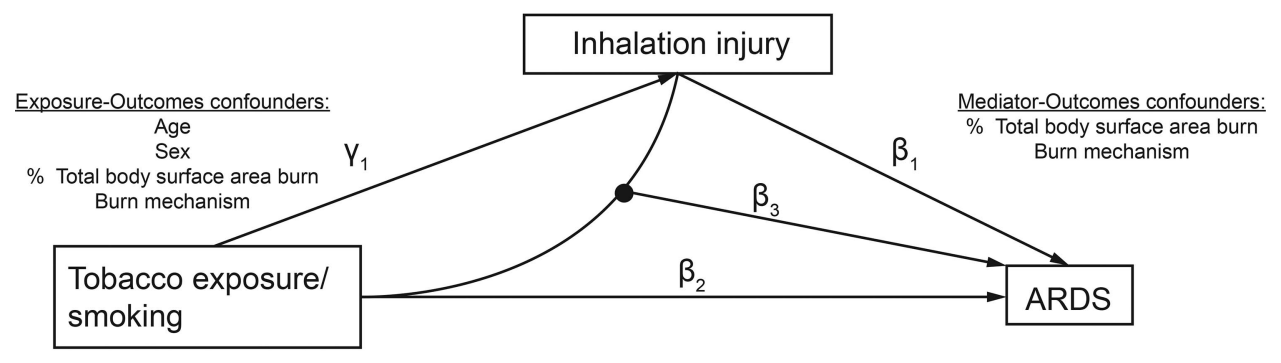

\begin{tabular}{|c|c|c|c|}
\hline Effects & $\begin{array}{l}\text { Odds } \\
\text { Ratio }\end{array}$ & $95 \% \mathrm{Cl}$ & $P$ \\
\hline $\begin{array}{l}\text { Effect of tobacco on ARDS NOT allowing for tobacco and } \\
\text { inhalation injury interaction: } \\
\text { Pure direct effect }\left(\beta_{2}+\beta_{3 \gamma 0}\right)\end{array}$ & 1.72 & $1.07-2.77$ & .02 \\
\hline $\begin{array}{l}\text { Effect of tobacco on ARDS allowing for tobacco and } \\
\text { inhalation injury interaction: } \\
\text { Total direct effect }\left(\beta_{2}+\beta_{3 \gamma 0}+\beta_{3 \gamma 1}\right)\end{array}$ & 1.63 & $0.917-2.92$ & .10 \\
\hline $\begin{array}{l}\text { Effect of tobacco on ARDS through inhalation injury as a } \\
\text { mediator only: } \\
\text { Total indirect effect }\left(\beta_{1 \mathrm{y} 1}\right)\end{array}$ & 1.61 & $1.25-2.07$ & $<.001$ \\
\hline $\begin{array}{l}\text { Effect of tobacco on ARDS through inhalation injury as a } \\
\text { mediator and allowing for interaction: } \\
\text { Total indirect effect }\left(\beta_{1 \mathrm{y} 1}+\beta_{3 \gamma 1}\right)\end{array}$ & 1.52 & $1.17-1.97$ & $<.001$ \\
\hline $\begin{array}{l}\text { Total effect*: } \\
\text { (Total indirect effect + pure indirect effect) }\end{array}$ & 2.62 & $1.47-4.69$ & $<.001$ \\
\hline
\end{tabular}

${ }^{*} 55.6 \%$ of total effect mediated by inhalation injury

Fig. 1. A mediation model with exposure-mediator interactions. The filled circle represents an interaction term consisting of the variables connected to it without arrowheads, in this case tobacco exposure and inhalation injury. $\beta_{1} \gamma_{1}=$ indirect effect; $\beta_{2}=$ direct effect; $\beta_{3}=$ exposure-mediator interaction; $\gamma_{0}=$ intercept. Effects in the model are shown above.

North Carolina). The institutional review board of Loyola University Chicago approved this study.

\section{Results}

A total of 2,485 subjects arrived at the burn center with a primary diagnosis for burn injury. The cohort case rate for development of ARDS was $6.8 \%(n=170)$. The majority of admissions for burn were total body surface area injury $<10 \%(n=2,008,80.8 \%)$, but $44.1 \%(n=75)$ of the ARDS cases occurred in this group, of which $54.7 \%$ $(n=42)$ had inhalation injury. The median (interquartile range) time to ARDS development was 1.7 (0.4-3.6) d. Inhalation injury with or without burn injuries occurred in $5.0 \%(n=125)$ of the cohort, and ARDS development occurred in $48.8 \%(n=83)$ of these subjects. Over $90 \%$ $(n=113)$ of the inhalation injury subjects with ARDS had at least moderate inhalation injury (abbreviated injury score $\geq 2$ ). Few subjects in the cohort had prior respiratory or cardiac disease (Table 1). Subjects with ARDS were older and had more comorbidities than their counterparts without ARDS. Burn injury characteristics were more severe in the ARDS group. These subjects habitually consumed more alcohol and had nearly 2-fold more tobacco use than those without ARDS (Table 1).

\section{Outcomes Between ARDS and Non-ARDS Patients}

The mean 28-d ICU-free days was lower in ARDS subjects than their non-ARDS counterparts (4.4 vs $20.5 \mathrm{~d}$, $P<.001)$, and as expected, the in-hospital case fatality rate in subjects with ARDS was much higher (25.3\% vs $2.2 \%, P<.001)$. In burn subjects with ARDS, the median $\mathrm{P}_{\mathrm{aO}_{2}} / \mathrm{F}_{\mathrm{IO}_{2}}$ was similar between tobacco and non-tobacco users (189.8 vs $186.2, P=.65$ ). Furthermore, the $28-\mathrm{d}$ ICU-free days and in-hospital death rate were similar between tobacco and non-tobacco users with ARDS $(P=.44$ and .25 , respectively).

\section{Association Between Tobacco Use, Inhalational Injury, and ARDS}

In multivariable logistic regression analysis adjusted for age, sex, burn mechanism, total body surface area injured, 
Tobacco Use, ARDS, AND InHALATION INJURY

Table 1. Subject Characteristics and Outcomes by ARDS Group

\begin{tabular}{|c|c|c|c|c|}
\hline Variable & Total $(N=2,485)$ & No ARDS $(n=2,315)$ & $\operatorname{ARDS}(n=170)$ & $P$ \\
\hline Age, median years (IQR) & $43(30-56)$ & $42(29-55)$ & $51(38-61)$ & $<.001$ \\
\hline BMI, median (IQR) & $27.3(23.9-31.5)$ & $27.2(23.7-31.4)$ & $28.6(25.4-32.4)$ & .02 \\
\hline Male sex, $n(\%)$ & $1,780(71.6)$ & $1,664(71.9)$ & $113(66.5)$ & .13 \\
\hline Race/ethnicity, $n(\%)$ & & & & $<.001$ \\
\hline Non-Hispanic black & $414(16.7)$ & $385(16.6)$ & $29(17.1)$ & \\
\hline Non-Hispanic white & $1,545(62.2)$ & $1,281(55.3)$ & $103(60.6)$ & \\
\hline Hispanic & $170(6.8)$ & $155(6.7)$ & $15(8.8)$ & \\
\hline Diabetes, $n(\%)$ & $268(10.8)$ & $237(10.2)$ & $31(18.2)$ & $<.001$ \\
\hline Hypertension, $n(\%)$ & $646(26.0)$ & $566(24.5)$ & $80(47.1)$ & $<.001$ \\
\hline Respiratory disease, ${ }^{*} n(\%)$ & $254(10.2)$ & $219(9.5)$ & $35(20.6)$ & $<.001$ \\
\hline Congestive heart failure, $n(\%)$ & $20(0.8)$ & $13(0.6)$ & $7(4.1)$ & $<.001$ \\
\hline Tobacco use, $n(\%)$ & $572(23.1)$ & $504(21.8)$ & $68(40.0)$ & $<.001$ \\
\hline $\mathrm{BAC}>0 \mathrm{mg} / \mathrm{dL}, n(\%)(n=925)$ & $121(13.1)$ & $130(16.2)$ & $36(29.8)$ & $<.001$ \\
\hline TBSA, median (IQR) \% & $2.3(0.9-6.0)$ & $2.1(0.8-5.1)$ & $17.1(6.2-38.5)$ & $<.001$ \\
\hline \multicolumn{5}{|l|}{ Burn mechanism, $n(\%)$} \\
\hline Chemical & $199(8.0)$ & $195(8.5)$ & $4(2.4)$ & \\
\hline Flame & $1,125(45.3)$ & $999(43.3)$ & $126(74.1)$ & \\
\hline Grease & $181(7.3)$ & $181(7.9)$ & $0(0)$ & $<.001$ \\
\hline Scald & $470(18.9)$ & $464(20.1)$ & $6(3.5)$ & \\
\hline Other $\dagger$ & $510(20.6)$ & $425(18.5)$ & $9(5.3)$ & \\
\hline Inhalation injury only, $n(\%)$ & $62(2.5)$ & $37(1.7)$ & $25(14.7)$ & $<.001$ \\
\hline Flame injury + inhalation injury, $n(\%)(n=1,125)$ & $92(8.2)$ & $32(2.8)$ & $60(5.3)$ & $<.001$ \\
\hline Inhalation injury score, $n(\%)$ & & & & $<.001$ \\
\hline 0 & $2,360(95.0)$ & $2,273(98.2)$ & $87(51.2)$ & \\
\hline $1-2$ & $69(2.8)$ & $31(1.3)$ & $38(22.4)$ & \\
\hline $3-4$ & $56(2.2)$ & $11(0.5)$ & $45(26.5)$ & \\
\hline Admission $\mathrm{SBP}<90, n(\%)$ & $513(20.6)$ & $361(3.9)$ & $152(89.4)$ & .02 \\
\hline \multicolumn{5}{|l|}{ Disposition, $n(\%)$} \\
\hline Home & $2,097(84.4)$ & $2,047(88.4)$ & $50(29.4)$ & $<.001$ \\
\hline Skilled nursing facility/in-patient rehabilitation & $156(6.4)$ & $121(5.2)$ & $35(20.6)$ & \\
\hline Long-term care hospital & $36(1.4)$ & $13(0.6)$ & $23(13.5)$ & \\
\hline In-hospital death & $97(3.9)$ & $54(2.3)$ & $43(25.4)$ & \\
\hline Other & 99 (3.9) & $80(3.5)$ & $19(11.1)$ & \\
\hline $\begin{array}{l}\text { * Asthma, COPD, pulmonary embolism, and other respiratory diseases } \\
\dagger \text { Includes contact, electrical, flash, friction, frostbite, lightning, radiati } \\
\text { † Includes against medical advice, transfer to another facility, hospice, } \\
\text { IQR = interquartile range } \\
\text { BMI = body mass index } \\
\text { BAC = blood alcohol concentration } \\
\text { TBSA = total body surface area injured } \\
\text { SBP }=\text { systolic blood pressure }\end{array}$ & $\begin{array}{l}\text { and unknown. } \\
\text { chiatry. }\end{array}$ & & & \\
\hline
\end{tabular}

and inhalation injury score (Fig. 2), the OR for the association between tobacco exposure and ARDS development was 1.84 (95\% CI 1.22-2.81, $P<.001$ ). Inhalation injury had the highest odds for ARDS development among the risk factors (OR 37.08, 95\% CI 22.97-59.86, $P<.001$ ), followed by total body surface area injured $\geq 33 \%$ (OR $12.92,95 \%$ CI $6.88-24.26, P<.001)$. An increasing inhalation injury score was associated with increasing risk for ARDS development $(P<.001)$. Inhalation injury also provided better discrimination for the development of ARDS than tobacco use, with a receiver operating characteristic area under the curve of 0.74 (95\% CI $0.70-0.77)$ versus 0.59 (95\% CI $0.55-0.63), P<.001$ (Fig. 3). In propensity score analysis $(n=1,130)$, the association between tobacco use and ARDS development remained significant, with an OR of 1.69 (95\% CI 1.04-2.75, $P=.034$ ) (see Fig. 2).

However, in the mediated model, the total direct effect of tobacco exposure on ARDS, including interaction between tobacco use and inhalation injury, was not significant (OR 1.63, 95\% CI $0.91-2.92, P=.10$ ). The pure indirect effect of tobacco exposure via inhalation injury as the mediator between tobacco use and ARDS development was significant (OR 1.61, 95\% CI 1.25-2.07, $P<.001$ ) 


\section{Multivariable Logistic Regression Model}

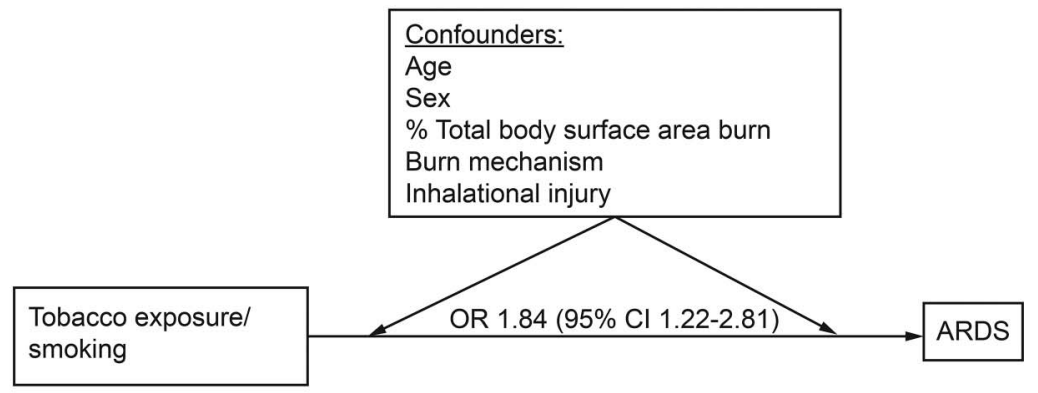

\section{Propensity Score Analysis Model}

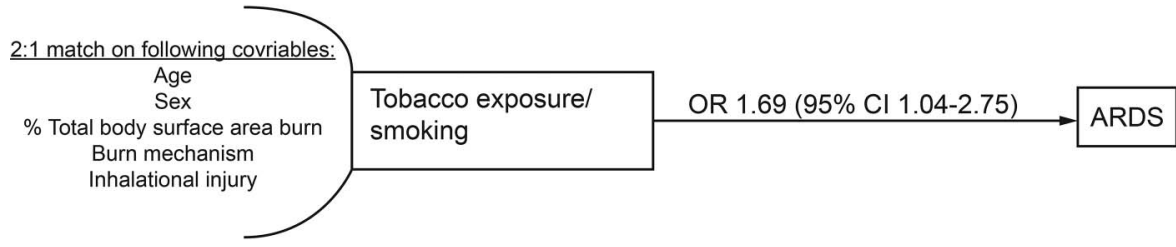

Fig. 2. Multivariable logistic regression with tobacco use as the independent variable and ARDS as the dependent variable. Age, sex, mechanism of burn, percentage of total body surface area injured, and inhalation injury are covariables in the model. Shown is propensity score analysis with conditional logistic regression after 2:1 matching of the covariables age, sex, percentage of total body surface area injured, burn mechanism, and inhalation injury between non-tobacco users and tobacco users for the association with ARDS.

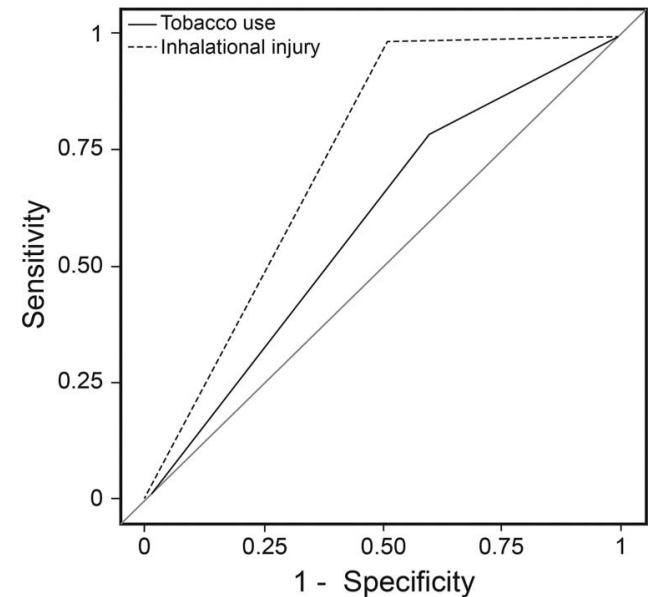

Fig. 3. Receiver operating characteristic curves comparing inhalation injury and tobacco use for discriminating ARDS development. Areas under the curve are 0.59 for tobacco use and 0.74 for inhalational injury.

(Fig. 1). The proportion of ARDS development mediated by inhalation injury in the total effect was $55.6 \%$. Comparison of all 3 models is shown in Figure 4.

\section{Analysis Stratified to Flame Injury Only}

In sub-analysis involving only flame injuries $(n=1,125)$, tobacco use had an increased OR for ARDS development in multivariable logistic regression (OR 2.01,

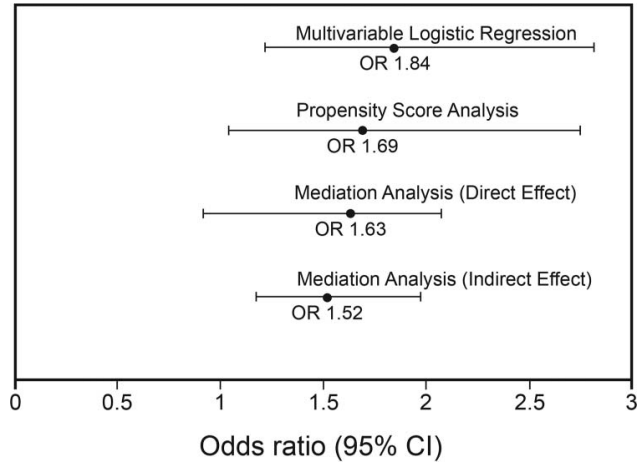

Fig. 4. Comparison of unmediated and mediated models for the relationship between tobacco exposure and ARDS development.

95\% CI 1.24-3.26, $P<.001)$, and inhalation injury continued to have the greatest OR $(20.17,95 \%$ CI 11.51 35.33, $P<.001)$ for development of ARDS. In mediation analysis, the total direct effect from tobacco use on ARDS development was significant (OR 2.83, 95\% CI 1.08-7.39, $P=.042)$, but the total indirect effect with inhalation injury as the mediator continued to have the strongest association (OR 1.74, 95\% CI 1.27-2.40, $P<.001$ ), and the proportion of ARDS development mediated by inhalation injury in the total effect was $57.0 \%$, similar to the analysis from the full cohort (Fig. 5). In sensitivity analysis, we assumed that sepsis occurred in $30 \%$ of ARDS cases versus $7 \%$ in non-ARDS cases, as reported in prior studies, ${ }^{23,24}$ and the case rate for ARDS in smokers versus 


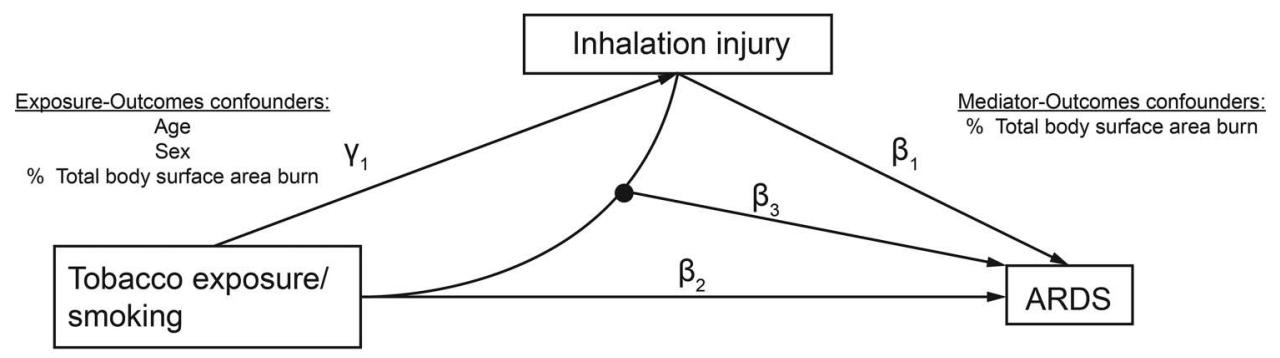

\begin{tabular}{|c|c|c|c|}
\hline Effects & $\begin{array}{l}\text { Odds } \\
\text { Ratio }\end{array}$ & $95 \% \mathrm{Cl}$ & $P$ \\
\hline $\begin{array}{l}\text { Effect of tobacco on ARDS NOT allowing for tobacco and } \\
\text { inhalation injury interaction: } \\
\text { Pure direct effect }\left(\beta_{2}+\beta_{3 \gamma 0}\right)\end{array}$ & 2.27 & $1.32-3.90$ & .01 \\
\hline $\begin{array}{l}\text { Effect of tobacco on ARDS allowing for tobacco and } \\
\text { inhalation injury interaction: } \\
\text { Total direct effect }\left(\beta_{2}+\beta_{3 y_{0}}+\beta_{3 y_{1}}\right)\end{array}$ & 2.83 & $1.08-7.39$ & .042 \\
\hline $\begin{array}{l}\text { Effect of tobacco on ARDS through inhalation injury as a } \\
\text { mediator only: } \\
\text { Total indirect effect }\left(\beta_{1 \mathrm{y} 1}\right)\end{array}$ & 1.58 & $1.20-2.10$ & $<.001$ \\
\hline $\begin{array}{l}\text { Effect of tobacco on ARDS through inhalation injury as a } \\
\text { mediator and allowing for interaction: } \\
\text { Total indirect effect }\left(\beta_{1 \mathrm{y} 1}+\beta_{3 \mathrm{y} 1}\right)\end{array}$ & 1.74 & $1.27-2.41$ & $<.001$ \\
\hline $\begin{array}{l}\text { Total effect*: } \\
\text { (Total indirect effect }+ \text { pure indirect effect) }\end{array}$ & 3.96 & $2.00-7.83$ & $<.001$ \\
\hline
\end{tabular}

*57.0\% of total effect mediated by inhalation injury

Fig. 5. A mediation model with exposure-mediator interactions for the analysis stratified to flame injury only $(n=1,125)$. The filled circle represents an interaction term consisting of the variables connected to it without arrowheads, in this case tobacco exposure and inhalation injury. $\beta_{1} \gamma_{1}=$ indirect effect; $\beta_{2}=$ direct effect; $\beta_{3}=$ exposure-mediator interaction; $\gamma_{0}=$ intercept. Effects in the model are shown above.

non-smokers in our cohort was $40 \%$ versus $22 \%$. The total direct effect remained insignificant, with an OR of 2.11 (95\% CI 0.81-6.84).

\section{Discussion}

Our data using mediation analysis demonstrated most of the association between tobacco use and ARDS development to be mediated through inhalation injury with limited contribution from tobacco use itself. Commonly used statistical approaches like multivariable logistic regression and propensity score analysis are limited in their ability to account for intermediate outcomes (ie, inhalation injury) in determining associations between exposure (ie, tobacco use) and outcomes of interest (ie, ARDS). Results from these models suggest that tobacco use is an important risk factor for ARDS development in burn-injured adults. In contrast, mediation analysis allows for intermediate outcomes in analyzing the direct and indirect associations between tobacco use, inhalation injury, and ARDS and therefore more accurately approximates the risk from tobacco use before burn/inhalation injury.
This study cohort had few comorbidities and experienced minor burns that probably contributed to the low case rate for ARDS. Prior studies in trauma cohorts with inclusion of all encounters have demonstrated similar rates of ARDS development between 5 and 10\%.7,23,24 Similar rates from the National In-Patient Sample and the National Burn Repository of in-patient admissions for burn with concomitant inhalation injury were noted around 5\%.6 However, our study revealed that nearly half of the cohort suffering from inhalation injury developed ARDS, much higher than the $20 \%$ reported in previous smaller studies. ${ }^{9,25}$ A prospective multi-center observational study with ARDS cases reviewed by pulmonary and critical care physicians confirmed a similar rate to this study at $43 \% .{ }^{26}$ Despite a lower case rate, our burn-ARDS phenotype was older, were characterized by increased comorbidities, and had more substance use than the non-ARDS group, similar to ARDS phenotypes described previously. ${ }^{23,27}$

The prevalence of smoking in the United States population was estimated at $16.8 \%$ in 2015 ; our cohort of burn subjects had higher rates than the general United States population, with tobacco use present in approximately $25 \%$. 
Epidemiologic studies have confirmed the increased risk for burn injury from cigarette smoking, and our cohort had an increased OR for inhalation injury in the mediated model. Tobacco use also had an increased odds risk for ARDS development, which was previously described in a trauma cohort. ${ }^{11}$ In some cases, tobacco use may have contributed to the injury as part of the indirect pathway toward ARDS, whereas in other cases, tobacco use itself potentially modulated the innate immune response and inflammatory milieu of the lung independent of inhalation injury and consequently engendered ARDS. Experimental studies have demonstrated that active smoking leads to pathophysiological changes similar to those in ARDS, including increased lung epithelial permeability, endothelial injury, and dysregulated platelet function. ${ }^{28-31}$ However, multiple outcomes in our subjects with ARDS were not affected by tobacco use, including $\mathrm{P}_{\mathrm{aO}} / \mathrm{F}_{\mathrm{IO}_{2}}$ ratio, 28-d ICU-free days, and in-hospital fatality rate. Although multivariable logistic regression and propensity score analysis was remarkable for a positive association between tobacco use and ARDS development, mediation analysis indicated that the indirect effect of tobacco use was most important via inhalation injury. On the OR scale, $56 \%$ of the increased risk was due to inhalation injury as the mediator. This was observed despite interactions between tobacco use and inhalation injury as well as adjustment for total body surface area injured, mechanism of injury, and subject characteristics. Because tobacco use may both predispose individuals to injury and influence the severity of injury, traditional adjustment for it probably introduced an overadjustment bias in multivariable logistic regression and propensity score analysis. ${ }^{22,32-34}$ Mediation analysis more accurately reflects the important exposures for ARDS and indicated that the majority of the effect from smoking on ARDS development was via inhalation injury as the mediator.

The results did vary when the analysis was stratified by flame injury to remove other burn mechanisms that probably do not suffer inhalation injury (ie, electrical, scald, contact). In the flame injury group, which comprised the largest subgroup in the cohort, the direct effect that included the effect of tobacco use on ARDS allowing for tobacco use and inhalation injury interaction was significant. However, like the analysis in the full cohort, the relationship remained the strongest via the indirect effect, which demonstrated a highly significant $P$ value. Furthermore, $57 \%$ of the total effect was mediated by inhalation injury, similar to the full cohort. Nevertheless, it is possible that tobacco use had a significant direct effect in the flame-only group because patients injured from combustible tobacco products typically occur in settings like a house fire and present with flame mechanisms and inhalation injury, making the injuries more difficult to parse out and the interaction stronger.
Inhalation injury was an overwhelming predictor for ARDS, as characterized by better sensitivity and specificity for ARDS development and a dose-dependent increase in the OR for ARDS development with higher grades of inhalation injury. Several mechanisms from experimental studies of inhalation injury in both humans and animals established a dysregulated innate immune response in the airway. ${ }^{35-37}$ Increased permeability of the microvasculature in the setting of a 20-fold increased bronchial blood flow, intra-airway coagulation and fibrin deposition, cellular debris, obstructive airway casts, large volume resuscitation, and fibrinocellular exudate all contribute to ventilation and perfusion mismatching with maldistribution of alveolar volumes causing both atelectasis and barotrauma. ${ }^{38-43}$ Qualified chest radiograph reports using key words for ARDS definition may not discern between cardiogenic and proteinaceous pulmonary edema. Subjects with ARDS had larger percentage of total body surface area injured and worse inhalational injuries, so they probably received larger volumes of crystalloid resuscitation, probably contributing to false positives for ARDS cases. Inter-observer variability in chest radiograph interpretation commonly occurs in characterizing cardiogenic versus non-cardiogenic pulmonary edema; however, it is expected that hydrostatic edema in the form of cardiac failure or fluid overload may coexist with ARDS, and the Berlin definition accepts respiratory failure not fully explained by cardiac failure or fluid overload for ARDS definition. Although the false positive rates were low, they probably contributed to variability in our results.

The strengths of this study were its large size, verified injury characteristics, and validated ARDS diagnoses that are more robust than another large burn study based on administrative data alone. ${ }^{25}$ Several limitations were present in this study including its retrospective and single center design. The use of administrative data places limitations on the quality of the data, with missing data and biases that could not be accounted for. Although the ARDS screening system was previously validated, prior studies indicated that inter-observer reliability is poor between radiologists for pulmonary edema versus ARDS, ${ }^{44}$ so misclassification bias may have occurred. Tobacco use was assessed by administrative data alone, whereas prior studies confirmed tobacco use with biomarkers, including cotinine. ${ }^{11,12}$ Chronic alcohol use is an important risk factor for ARDS, ${ }^{19}$ and co-substance use between tobacco and alcohol are common; however, we did not have comprehensive data on alcohol use to examine these effects. Data for ventilator settings were not collected and could not be assessed in the evaluation for ARDS development. The practice behaviors of burn critical care physicians probably varied between practitioners, and the use of lung-protective ventilation strategies was not standardized. In addition, our study was limited by lack of data for sepsis and 
other infectious complications that have been reported to occur in nearly a third of critically ill burn patients. ${ }^{45}$ However, sensitivity analysis was performed to address this limitation. Our results did not change with the inclusion of sepsis as an unmeasured confounder.

\section{Conclusions}

Tobacco use constitutes an important risk for burn injury and is a common problem in burn-injured patients. Although tobacco use increased the odds of ARDS, the large majority of the risk for ARDS derives from the burn injury alone and, in particular, inhalation injury. In a subgroup of burn subjects with flame mechanism, tobacco had a significant direct effect on the development of ARDS, but the majority of the effect was still mediated through inhalation injury, similar to the full cohort. Prospective clinical studies are needed to better examine the effect of inhalation injury over other risk factors so that clinicians may focus care to attenuate the strongest determinants for ARDS development.

\section{ACKNOWLEDGMENTS}

We thank the clinical research nurse Peggie Conrad for managing the burn registry and appreciate the Loyola Clinical Research Database leadership of Ron Price and programming by Susan Zelisko.

\section{REFERENCES}

1. Bessey PQ, Phillips BD, Lentz CW, Edelman LS, Faraklas I, Finocchiaro MA, et al. Synopsis of the 2013 annual report of the national burn repository. J Burn Care Res 2014;35(Suppl 2):S218S234.

2. McGuire A. Cigarettes and fire deaths. N Y State J Med 1983; 83(13):1296-1298.

3. Birky MM, Clarke FB. Inhalation of toxic products from fires. Bull N Y Acad Med 1981;57(10):997-1013.

4. Leistikow BN, Martin DC, Milano CE. Fire injuries, disasters, and costs from cigarettes and cigarette lights: a global overview. Prev Med 2000;31(2 Pt 1):91-99.

5. Sacks JJ, Nelson DE. Smoking and injuries: an overview. Prev Med 1994;23(4):515-520.

6. Veeravagu A, Yoon BC, Jiang B, Carvalho CM, Rincon F, Maltenfort $\mathrm{M}$, et al. National trends in burn and inhalation injury in burn patients: results of analysis of the nationwide inpatient sample database. J Burn Care Res 2015;36(2):258-265.

7. Trillo-Alvarez C, Cartin-Ceba R, Kor DJ, Kojicic M, Kashyap R, Thakur S, et al. Acute lung injury prediction score: derivation and validation in a population-based sample. Eur Respir J 2011;37(3): 604-609.

8. Gajic O, Dabbagh O, Park PK, Adesanya A, Chang SY, Hou P, et al. Early identification of patients at risk of acute lung injury: evaluation of lung injury prediction score in a multicenter cohort study. Am J Respir Crit Care Med 2011;183(4):462-470.

9. Belenkiy SM, Buel AR, Cannon JW, Sine CR, Aden JK, Henderson $\mathrm{JL}$, et al. Acute respiratory distress syndrome in wartime military burns: application of the Berlin criteria. J Trauma Acute Care Surg 2014;76(3):821-827.
10. Cartotto R, Li Z, Hanna S, Spano S, Wood D, Chung K, Camacho F. The acute respiratory distress syndrome (ARDS) in mechanically ventilated burn patients: an analysis of risk factors, clinical features, and outcomes using the Berlin ARDS definition. Burns 2016;42(7): 1423-1432.

11. Calfee CS, Matthay MA, Eisner MD, Benowitz N, Call M, Pittet JF, Cohen MJ. Active and passive cigarette smoking and acute lung injury after severe blunt trauma. Am J Respir Crit Care Med 2011; 183(12):1660-1665.

12. Calfee CS, Matthay MA, Kangelaris KN, Siew ED, Janz DR, Bernard GR, et al. Cigarette smoke exposure and the acute respiratory distress syndrome. Crit Care Med 2015;43(9):1790-1797.

13. Hsieh SJ, Zhuo H, Benowitz NL, Thompson BT, Liu KD, Matthay MA, et al. Prevalence and impact of active and passive cigarette smoking in acute respiratory distress syndrome. Crit Care Med 2014; 42(9):2058-2068.

14. Amato MB, Meade MO, Slutsky AS, Brochard L, Costa EL, Schoenfeld DA, et al. Driving pressure and survival in the acute respiratory distress syndrome. N Engl J Med 2015;372(8):747-755.

15. Mosier MJ, Pham TN, Park DR, Simmons J, Klein MB, Gibran NS. Predictive value of bronchoscopy in assessing the severity of inhalation injury. J Burn Care Res 2012;33(1):65-73.

16. ARDS Definition Task Force, Ranieri VM, Rubenfeld GD, Thompson BT, Ferguson ND, Caldwell E, et al. Acute respiratory distress syndrome: the Berlin definition. JAMA 2012;307(23):2526-2533.

17. Bernard GR, Artigas A, Brigham KL, Carlet J, Falke K, Hudson L, et al. The American-European Consensus Conference on ARDS: definitions, mechanisms, relevant outcomes, and clinical trial coordination. Am J Respir Crit Care Med 1994;149(3 Pt 1):818-824.

18. Afshar M, Smith GS, Terrin ML, Barrett M, Lissauer ME, Mansoor $\mathrm{S}$, et al. Blood alcohol content, injury severity, and adult respiratory distress syndrome. J Trauma Acute Care Surg 2014;76(6):1447-1455.

19. Moss M, Parsons PE, Steinberg KP, Hudson LD, Guidot DM, Burnham EL, et al. Chronic alcohol abuse is associated with an increased incidence of acute respiratory distress syndrome and severity of multiple organ dysfunction in patients with septic shock. Crit Care Med 2003;31(3):869-877.

20. Moss M, Guidot DM, Steinberg KP, Duhon GF, Treece P, Wolken $\mathrm{R}$, et al. Diabetic patients have a decreased incidence of acute respiratory distress syndrome. Crit Care Med 2000;28(7):2187-2192.

21. Cooke CR, Erickson SE, Watkins TR, Matthay MA, Hudson LD, Rubenfeld GD. Age-, sex-, and race-based differences among patients enrolled versus not enrolled in acute lung injury clinical trials. Crit Care Med 2010;38(6):1450-1457.

22. VanderWeele TJ. Mediation analysis: a practitioner's guide. Annu Rev Public Health 2016;37:17-32.

23. Bellani G, Laffey JG, Pham T, Fan E, Brochard L, Esteban A, et al. Epidemiology, patterns of care, and mortality for patients with acute respiratory distress syndrome in intensive care units in 50 countries JAMA 2016;315(8):788-800.

24. Rubenfeld GD, Caldwell E, Peabody E, Weaver J, Martin DP, Neff $\mathrm{M}$, et al. Incidence and outcomes of acute lung injury. N Engl J Med 2005;353(16):1685-1693.

25. Kadri SS, Miller AC, Hohmann S, Bonne S, Nielsen C, Wells C, et al. Risk factors for in-hospital mortality in smoke inhalationassociated acute lung injury: Data from 68 United States hospitals. Chest 2016;150(6):1260-1268.

26. Afshar M, Albright JM, Conrad P, Mosier MJ, Cooper RC, Burnham EL, Kovacs EJ. Acute respiratory distress syndrome in burn injured adults: utility of risk factors and biomarkers for identifying at-risk patients. In: Denver, Colorado: American Thoracic Society; 2015.

27. Calfee CS, Eisner MD, Ware LB, Thompson BT, Parsons PE, Wheeler AP, et al. Trauma-associated lung injury differs clinically and bio- 


\section{Tobacco Use, ARDS, AND InHALATION INJURY}

logically from acute lung injury due to other clinical disorders. Crit Care Med 2007;35(10):2243-2250.

28. Fernández JA, Gruber A, Heeb MJ, Griffin JH. Protein C pathway impairment in nonsymptomatic cigarette smokers. Blood Cells Mol Dis 2002;29(1):73-82.

29. Jones JG, Minty BD, Lawler P, Hulands G, Crawley JC, Veall N. Increased alveolar epithelial permeability in cigarette smokers. Lancet 1980;1(8159):66-68.

30. Li XY, Rahman I, Donaldson K, MacNee W. Mechanisms of cigarette smoke induced increased airspace permeability. Thorax 1996; 51(5):465-471.

31. Mason GR, Uszler JM, Effros RM, Reid E. Rapidly reversible alterations of pulmonary epithelial permeability induced by smoking. Chest 1983;83(1):6-11.

32. Schisterman EF, Cole SR, Platt RW. Overadjustment bias and unnecessary adjustment in epidemiologic studies. Epidemiology 2009; 20(4):488-495.

33. Vanderweele TJ, Vansteelandt S. Conceptual issues concerning mediation, interventions and composition. Statistics Interface 2009;2: 457-468.

34. VanderWeele TJ. On the relative nature of overadjustment and unnecessary adjustment. Epidemiology 2009;20(4):496-499.

35. Davis CS, Esposito TJ, Palladino-Davis AG, Rychlik K, Schermer CR, Gamelli RL, Kovacs EJ. Implications of alcohol intoxication at the time of burn and smoke inhalation injury: an epidemiologic and clinical analysis. J Burn Care Res 2013;34(1):120-126.

36. Albright JM, Davis CS, Bird MD, Ramirez L, Kim H, Burnham EL, et al. The acute pulmonary inflammatory response to the graded severity of smoke inhalation injury. Crit Care Med 2012;40(4):11131121.
37. Davis CS, Janus SE, Mosier MJ, Carter SR, Gibbs JT, Ramirez L, et al. Inhalation injury severity and systemic immune perturbations in burned adults. Ann Surg 2013;257(6):1137-1146.

38. Morita N, Enkhbaatar P, Maybauer DM, Maybauer MO, Westphal M, Murakami K, et al. Impact of bronchial circulation on bronchial exudates following combined burn and smoke inhalation injury in sheep. Burns 2011;37(3):465-473.

39. Jacob S, Kraft R, Zhu Y, Jacob RK, Herndon DN, Traber DL, et al. Acute secretory cell toxicity and epithelial exfoliation after smoke inhalation injury in sheep: an electron and light microscopic study. Toxicol Mech Methods 2010;20(8):504-509.

40. Hamahata A, Enkhbaatar P, Sakurai H, Nozaki M, Traber DL. Effect of ablated bronchial blood flow on survival rate and pulmonary function after burn and smoke inhalation in sheep. Burns 2009;35(6): 802-810.

41. Cox RA, Mlcak RP, Chinkes DL, Jacob S, Enkhbaatar P, Jaso J, et al. Upper airway mucus deposition in lung tissue of burn trauma victims. Shock 2008;29(3):356-361.

42. Murakami K, Traber DL. Pathophysiological basis of smoke inhalation injury. News Physiol Sci 2003;18:125-129.

43. Enkhbaatar P, Traber DL. Pathophysiology of acute lung injury in combined burn and smoke inhalation injury. Clin Sci 2004;107(2): 137-143.

44. Badgett RG, Mulrow CD, Otto PM, Ramírez G. How well can the chest radiograph diagnose left ventricular dysfunction? J Gen Intern Med 1996;11(10):625-634.

45. Lentz CW, Bessey PW, Phillips BD, Edelman LS, Farakias I, Finocchiaro MA, et al. 2014 National Burn Repository: report of data from 2004-2013. Chicago, Illinois: American Burn Association; 2014. 